Revue musicale OICRM

\title{
Le modèle, une voie vers la professionnalisation. L'exemple de Yuli Turovsky et de ses étudiants
}

\section{Florence Leyssieux}

Volume 1, numéro 1, 2012

Profession musicale au Québec

URI : https://id.erudit.org/iderudit/1055855ar

DOI : https://doi.org/10.7202/1055855ar

Aller au sommaire du numéro

\section{Éditeur(s)}

OICRM

ISSN

2368-7061 (numérique)

Découvrir la revue

Citer cet article

Leyssieux, F. (2012). Le modèle, une voie vers la professionnalisation. L'exemple de Yuli Turovsky et de ses étudiants. Revue musicale OICRM, 1(1), 1-19. https://doi.org/10.7202/1055855ar
Résumé de l'article

Basé sur une enquête réalisée auprès d'étudiants en interprétation de la Faculté de musique de l'Université de Montréal, cet article traite de la complexité du passage entre le monde de l'apprentissage et le monde professionnel chez les jeunes interprètes. L'absence de liens institutionnels entre la formation et la professionnalisation, ainsi que l'hétérogénéité des filières d'accès au monde du travail génèrent, pour les jeunes musiciens, des parcours d'insertion professionnelle placés sous l'égide de l'incertitude et de l'imprévisible. Cependant, l'analyse des données recueillies permet d'observer que le lien institutionnel manquant est remplacé par un lien individuel. On peut alors identifier le rôle que joue le professeur dans la construction d'un lien tant virtuel qu'effectif pour les jeunes interprètes en processus de professionnalisation. Car l'enseignant se positionne non seulement comme un maître où s'exerce un modèle de référence, mais aussi comme le "passeur " entre le monde de l'apprentissage et le monde professionnel. La notoriété du professeur et les démarches qu'il entreprend pour favoriser les expériences de scène de ses élèves ont des retombées importantes sur leurs conditions de professionnalisation. Dans cette optique, les actions menées par Yuli Turovsky, professeur à la Faculté de musique de l'Université de Montréal, soliste et chef d'orchestre, sont significatives. En s'appuyant sur la stabilité de modèles reconnus (orchestre de chambre et école stylistique d'interprétation), il crée pour ses étudiants des voies d'accès au monde du travail. Le modèle agit alors comme un outil pertinent d'intégration professionnelle et permet de contrecarrer partiellement l'indétermination liée à la réussite d'une carrière artistique.
Ce document est protégé par la loi sur le droit d'auteur. L'utilisation des services d'Érudit (y compris la reproduction) est assujettie à sa politique d'utilisation que vous pouvez consulter en ligne.

https://apropos.erudit.org/fr/usagers/politique-dutilisation/ 


\title{
Le modèle, une voie vers la professionnalisation. L'exemple de Yuli Turovsky et de ses étudiants
}

\author{
Florence Leyssieux
}

\begin{abstract}
Résumé
Basé sur une enquête réalisée auprès d'étudiants en interprétation de la Faculté de musique de l'Université de Montréal, cet article traite de la complexité du passage entre le monde de l'apprentissage et le monde professionnel chez les jeunes interprètes. L'absence de liens institutionnels entre la formation et la professionnalisation, ainsi que l'hétérogénéité des filières d'accès au monde du travail génèrent, pour les jeunes musiciens, des parcours d'insertion professionnelle placés sous l'égide de l'incertitude et de l'imprévisible. Cependant, l'analyse des données recueillies permet d'observer que le lien institutionnel manquant est remplacé par un lien individuel. On peut alors identifier le rôle que joue le professeur dans la construction d'un lien tant virtuel qu'effectif pour les jeunes interprètes en processus de professionnalisation. Car l'enseignant se positionne non seulement comme un maître où s'exerce un modèle de référence, mais aussi comme le "passeur » entre le monde de l'apprentissage et le monde professionnel. La notoriété du professeur et les démarches qu'il entreprend pour favoriser les expériences de scène de ses élèves ont des retombées importantes sur leurs conditions de professionnalisation. Dans cette optique, les actions menées par Yuli Turovsky, professeur à la Faculté de musique de l'Université de Montréal, soliste et chef d'orchestre, sont significatives. En s'appuyant sur la stabilité de modèles reconnus (orchestre de chambre et école stylistique d'interprétation), il crée pour ses étudiants des voies d'accès au monde du travail. Le modèle agit alors comme un outil pertinent d'intégration professionnelle et permet de contrecarrer partiellement l'indétermination liée à la réussite d'une carrière artistique.
\end{abstract}

Mots clés : apprentissage ; interprète ; lien ; mentor ; modèle ; professionnalisation.

\begin{abstract}
Based on a survey among students in music performance of the Université de Montréal's Faculty of Music, this article discusses the complexity of the passage between learning and professional worlds for young performers. The absence of institutional links between training and professionalization as well as the heterogeneity of access to the working world create, for young musicians, uncertainty and unpredictability about professional integration. However, the analysis of the collected data reveals that the missing institutional link is replaced by an individual link. We can then identify the role
\end{abstract}


that the teacher plays in building a virtual and an effective link for young performers in the professionalization process. Because the teacher positions his/herself not only as a master in which a reference model is exerted, but also as the "ferryman" between learning and professional worlds. The teacher's reputation and his/her endeavours to promote the stage experiences of his/her students have a significant impact on their conditions of professionalization. In this context, the actions of Yuli Turovsky, a professor at the Université de Montréal's Faculty of Music, soloist and conductor, are significant. Based on the stability of recognized models (chamber orchestra and stylistic school of performance), he creates pathways to the working world for his students. The model then acts as a relevant tool for professional integration and partially prevents the uncertainty associated with the success of an artistic career.

Keywords: learning; link; mentor; model; performer; professionalization.

Pour les jeunes interprètes évoluant dans le domaine de la musique classique, le passage de l'état d'apprenti à celui de professionnel se réclame plus de cheminements singuliers et individualisés que de trajectoires balisées et normées ${ }^{1}$. On peut identifier deux principales raisons à cette situation. D'une part, les filières d'accès au milieu musical professionnel qui, régies par les principes d'incertitude et de concurrence, se caractérisent par leur manque de formalité et leur autorégulation par la profession ${ }^{2}$. D'autre part, l'insuffisance du rôle joué par les institutions supérieures d'enseignement musical dans l'insertion professionnelle des jeunes interprètes, comparativement à celui que peuvent jouer, par exemple, les écoles d'ingénieurs ou celles des professions médicales pour leurs futurs candidats à la profession.

Les institutions d'enseignement musical représentent des lieux protégés où tout est mis à la disposition des étudiants, où la compétition existe, mais reste limitée et plus facile à gérer et à maîtriser que dans le monde du travail, car les concurrents sont connus et se côtoient régulièrement. Généralement, les contacts s'opérant avec le milieu musical professionnel ne s'inscrivent pas dans le cursus institutionnel d'enseignement, mais découlent plutôt de démarches individuelles. Ainsi, pour les instrumentistes, les expériences de scène se font principalement au sein del'institution, et seules des initiatives personnelles (enseignant ou étudiant) permettent de sortir de ce cadre. Dans cet environnement relativement protecteur - bien que l'on observe de plus en plus de tentatives d'ajustement des programmes d'enseignement à la réalité professionnelle ${ }^{3}-$, la formation académique semble limitée par rapport aux conditions d'exercice du métier ${ }^{4}$.

1 Cette problématique est développée par Pierre-Michel Menger (2009) dans son livre Le travail créateur.

2 Ce que Pierre François résume ainsi : «Les musiciens professionnels ont mis en place des modalités de fonctionnement, institutionnelles ou informelles, qui permettent à la profession de s'autoréguler partiellement » (François 2004, p. 600).

3 Par exemple, la Faculté de musique de l'Université de Montréal offre un cours intitulé « Le métier de musicien ». Le cours vise à former les étudiants (interprètes, compositeurs ou musicologues) par rapport aux divers aspects pratiques et théoriques de la carrière professionnelle (demandes de subvention, droits d'auteurs, dossiers de presse, agences d'artistes, communications, etc.).

4 C'est une constatation que j'ai pu observer (au Québec et en France) durant mon parcours et qui était 
L'obtention d'un diplôme d'études supérieures, et éventuellement celle de prix internationaux, confère une crédibilité aux jeunes musiciens et fait figure de balise auprès des futurs employeurs. Toutefois, malgré les visées professionnelles de formation des conservatoires et des universités ${ }^{5}$, malgré la qualité de l'enseignement dispensé et le souci constant de "mettre au monde " des musiciens de plus en plus performants et compétitifs, les diplômes, le temps et l'énergie investis ne sont pas toujours conditionnels à l'obtention d'une situation professionnelle équivalente à la formation reçue, ni aux attentes et aux ambitions de l'apprenant. La forte concurrence opérant dans un marché assez étroit réduit les possibilités de réussite de carrière ou d'obtention de postes stables dans les sociétés musicales et les institutions d'enseignement. Quant aux carrières de soliste, leur réussite est l'aboutissement d'un parcours très sélectif, où les compétences exigées (musicales, techniques, personnelles) se situent à un niveau très élevé.

Si certaines conditions d'exercice du métier permettent aux interprètes d'obtenir socialement le statut de professionnels (institutions d'enseignement musical, orchestres, maisons d'opéra), la pratique musicale indépendante, qu'on peut associer aux professions libérales, n'étant ni normée, ni régie par un ordre ${ }^{6}$, rend délicate l'évaluation sociale du niveau de professionnalisation de l'interprète, si ce n'est par la communauté musicienne ${ }^{7}$. Or, dans les milieux artistiques, la reconnaissance professionnelle et sociale ne repose pas uniquement sur les compétences et les diplômes du musicien : elle est aussi subordonnée à une évaluation sociale. Conséquente aux jugements de valeur d'autrui, elle est soumise à la versatilité des goûts du public, à l'impact et à l'influence des médias et des critiques, à l'intégrité et à la sincérité du jugement des pairs, et à la capacité qu'a l'interprète de développer des relations interpersonnelles de qualité, d'établir des réseaux, de saisir des opportunités.

On saisit mieux alors les spécificités et l'hétérogénéité des parcours d'intégration professionnelle qui dépendent en grande partie de critères esthétiques, subjectifs, évènementiels et relationnels.

émise autant par les étudiants que par les enseignants, entre autres à la Faculté de musique de l'Université de Montréal.

5 Il faut toutefois faire une distinction entre l'enseignement dispensé dans les conservatoires, plus axé sur la pratique instrumentale (savoir-faire) et celui dispensé dans les universités où les disciplines théoriques occupent une place plus importante (savoir). À ce sujet, voir l'article de Johannella Tafuri, « La formation musicale supérieure en Europe et en Amérique du Nord » (Tafuri 2004).

6 Il existe au Québec un regroupement de musiciens professionnels, la Guilde des musiciens et musiciennes du Québec. Il s'agit d'un syndicat ayant pour mission de « représenter et défendre les intérêts moraux, sociaux et économiques des musiciens professionnels afin que leur apport à la société soit reconnu à sa juste valeur. » Voir : www.gmmq.com/fr (consulté le 17 février 2012). Les chanteurs, quant à eux, sont représentés par l'Union des artistes, le syndicat professionnel qui défend également les acteurs, les animateurs et les danseurs. Voir : https://uda.ca (consulté le 17 février 2012).

$7 \quad$ Pour plusieurs organismes québécois de soutien à la création et à la diffusion des arts, dont le Conseil des arts et des lettres du Québec (CALQ), un des critères de définition d'un artiste professionnel est d'avoir obtenu " une reconnaissance de ses pairs ». Voir : www.calq.gouv.qc.ca/artistes/musique.htm (consulté le 6 juin 2012). 
En dépit de cette réalité, les marchés artistiques, bien que placés sous l'égide de l'imprévisible et de l'aléatoire (Menger 2005, p. 44), exercent sur les candidats au métier de musicien une attraction particulière qui les incite à tenter leur chance, misant davantage sur leur détermination et leurs capacités que sur une connaissance réelle des conditions de travail et d'insertion au milieu professionnel.

Dans ce contexte, s'il n'existe pas de lien institutionnel entre l'acte de formation et l'acte de professionnalisation, comment s'opère le passage entre l'apprentissage et l'exercice du métier ? Existe-t-il une autre forme de lien, tacite, contribuant à l'insertion professionnelle des jeunes musiciens ? Si ce lien existe, quelle en est la nature et comment opère-t-il ?

Afin d'étudier la complexité de ce passage vers la professionnalisation musicale, une étude a été réalisée sur la base d'entrevues auprès d'étudiants en interprétation inscrits à la Faculté de musique de 1'Université de Montréa $1^{8}$. Les candidats interrogés, Zhou Fang ${ }^{9}$, violoncelliste d'origine chinoise, Julie Fuchs ${ }^{10}$, altiste française, et

8 Cette recherche avec des êtres humains a reçu l'aval du Comité plurifacultaire d'éthique de la recherche (CPÉR) de l'Université de Montréal dont relève la Faculté de musique.

9 Violoncelliste d'origine chinoise, arrivée au Québec en 1990. Issue d'une famille de musiciens (grand-père maternel et mère violoncellistes), elle a débuté l'étude du violoncelle vers cinq ans avec sa mère, puis a pris des cours privés avec un professeur avant de suivre un cursus institutionnel dans des classes à " horaires aménagés " (École secondaire Pierre Laporte puis études collégiales en musique au Cégep Marianopolis). Elle a ensuite poursuivi des études instrumentales universitaires. À partir de l'adolescence, elle a ressenti le besoin de prendre du recul par rapport à sa formation instrumentale. Ses études musicales ont été entrecoupées d'études en sciences et en gestion d'organismes culturels (HEC) qu'elle n'a pas terminées.

Son besoin de revenir constamment à la musique l'a incitée à faire un baccalauréat en interprétation à 1'Université McGill. Elle a ensuite étudié à Toronto puis à Calgary pour finalement revenir à Montréal, en 2010, et travailler avec Yuli Turovsky à l'Université de Montréal, où elle termine sa maîtrise en interprétation. Pour l'instant, ses expériences de scène se déroulent principalement dans le cadre de l'institution d'enseignement, avec quelques expériences de professionnalisation en dehors de la Faculté de musique.

Zhou Fang fait partie de l'Orchestre de l'Université de Montréal. Elle souhaiterait devenir musicienne d'orchestre - ce que lui ont toujours conseillé ses parents - et enseigner ; l'enseignement étant complémentaire à la pratique personnelle, car elle estime qu'il est important de savoir verbaliser ce que l'on expérimente en jouant ; c'est, selon elle, une source de réflexion.

Étant elle-même violoncelliste, la mère de Zhou Fang a suivi et conseillé sa fille durant ses premières années d'apprentissage, en orientant sa formation vers la pratique professionnelle du violoncelle, car elle souhaite que Zhou poursuive la tradition familiale et devienne violoncelliste dans un orchestre.

10 Altiste française, en seconde année de doctorat en interprétation avec Jutta Puchhammer-Sédillot (www.musique.umontreal.ca/personnel/puchhammer-sedillot j.html, consulté le 17 février 2012).

C'est suite à l'audition de Pierre et le loup (qu'elle écoutait régulièrement), que Julie émis le désir de faire de la musique. Elle a fait ses débuts à l'alto à l'âge de quatre ans, en France.

Après des études dans divers conservatoires régionaux, où elle a obtenu un Prix de perfectionnement, elle est entrée au Conservatoire national supérieur de musique et de danse de Lyon, dans la classe de Tasso Adamopoulos, altiste de renommée internationale qui a été la figure la plus marquante dans son itinéraire d'apprentissage. Elle a obtenu son diplôme de premier cycle parallèlement à une maîtrise en musicologie réalisée à la Faculté de musique de Lyon. En France, Julie a acquis une solide expérience de scène grâce aux nombreux remplacements qu'elle a faits dans différents orchestres nationaux et grâce également à son appartenance à l'Orchestre français des jeunes et à l'Orchestre des jeunes Gustav Mahler.

Suite à une rencontre avec Jutta Puchhammer-Sédillot, dans le cadre d'un stage d'été, Julie a décidé de terminer sa formation à la Faculté de musique de Montréal auprès de cette enseignante. Depuis son arrivée au Québec, en 2010, elle joue dans l'Orchestre 21 (www.wix.com/pbellomia/orchestre21, consulté 
Stéphane Tétreault ${ }^{11}$, violoncelliste québécois, sont tous les trois sous la tutelle de Yuli Turovsky ${ }^{12}$, violoncelliste d'origine russe, professeur à la Faculté de musique de

le 17 février 2012) et dans l'Orchestre Nouvelle Génération (remplacement d'une altiste en congé de maternité), tout en développant un projet de recherche autour du répertoire contemporain pour l'alto dans le cadre de son doctorat en interprétation.

Bien que non musiciens, ses parents respectent son choix et lui apportent un soutien et des encouragements constants. Par contre, ils n'ont pu être d'aucune aide sur le plan musical durant l'apprentissage des premières années, ce qui a conduit Julie à développer très tôt une méthode de travail personnelle par rapport aux demandes du professeur.

11 Violoncelliste québécois, terminant actuellement un baccalauréat en interprétation, à la Faculté de musique de l'Université de Montréal, dans la classe de Yuli Turovsky. Ses aptitudes artistiques ont été décelées par ses parents qui l'ont inscrit à l'École Face de Montréal, une école offrant un programme beaux-arts en complément du programme scolaire de base. C'est dans cette école que Stéphane a débuté l'étude du violoncelle à l'âge de sept ans. Après deux années d'études, il est devenu l'élève de Yuli Turovsky, avec qui il a étudié en cours privés durant neuf ans avant d'intégrer sa classe à la Faculté de musique de l'Université de Montréal. Le parcours de Stéphane Tétreault diffère un peu de celui que suivent généralement les jeunes étudiants en interprétation. Il a connu ses premières expériences de scène dès l'âge de neuf ans, grâce à ses premiers concerts et à sa participation à de nombreux concours nationaux et provinciaux, ce qui l'a aidé à grandir dans le milieu professionnel et à acquérir très tôt de la visibilité.

Âgé de 19 ans, son parcours musical est déjà très conséquent et brillant. Lauréat et finaliste de concours nationaux et internationaux, nommé Révélation Radio-Canada 2011-2012, il a amorcé une carrière prometteuse. Soliste invité de plusieurs orchestres (Orchestre Métropolitain, Orchestre de Malaisie, Orchestre I Musici de Montréal, Nouvel Ensemble Moderne), et de plusieurs festivals, son premier enregistrement, réalisé avec l'Orchestre symphonique de Québec dirigé par Fabien Gabel, est sorti en septembre 2012. Depuis janvier 2012, Stéphane joue le violoncelle «Comtesse de Stanlein, ex-Paganini » construit en 1707 par Stradivarius. Cet instrument lui a été prêté à vie par une mécène montréalaise, en reconnaissance de son talent exceptionnel.

Stéphane reçoit, depuis le début de sa formation, l'aide et le soutien continuels de ses parents qui s'investissent énormément dans la réussite de la carrière de leur fils. Son père, musicien amateur, a constamment suivi et aidé son fils dans la pratique journalière de son instrument, jusqu'à l'âge de onze ans, âge auquel Stéphane avait acquis suffisamment d'autonomie et de motivation pour travailler seul. Sa mère a contribué à lui constituer un réseau socioprofessionnel en restant en contact avec les organisateurs des concours régionaux auxquels il participait. C'est ainsi que s'est également constitué ce que Stéphane se plaît à nommer son «Fan club». Stéphane est membre de l'Orchestre Nouvelle Génération en tant que violoncelliste et chef d'orchestre.

12 Né en 1939, Yuli Turovsky a débuté le violoncelle à l'âge de sept ans à l'École centrale de musique de Moscou, puis au Conservatoire de cette même ville, sous la tutelle de Galina Kozolupova. Après l'obtention d'un doctorat en 1969, il remporte le premier prix du Concours de violoncelle d'URss et, l'année suivante, le second prix au Concours International Printemps de Prague. Devenu violoncelle solo de l'Orchestre de chambre de Moscou (dirigé par Rudolf Barshai), il a participé à de nombreux enregistrements et a fait plusieurs tournées internationales avec cet orchestre. Parallèlement à ses activités d'interprète, il a enseigné à l'École centrale de musique de Moscou où il était l'assistant de son ancien professeur, Galina Kozolupova.

Il a quitté 1'urss en 1976 et s'est installé à Montréal en 1977. Il a obtenu un poste de professeur de musique de chambre au Conservatoire de musique de Montréal la même année, poste qu'il a occupé jusqu'en 1985. Depuis 1981 il enseigne le violoncelle à la Faculté de musique de l'Université de Montréal. Il a poursuivi sa carrière d'interprète après son immigration au sein du Trio Borodine (avec Rostislav Dubinsky et Luba Eldina, de 1976 à 1993), du Duo Turovsky (avec son épouse Eleonora) et surtout en tant que chef d'orchestre, avec l'Orchestre de chambre I Musici de Montréal qu'il a fondé en 1983. De nombreux enregistrements (couronnés par plusieurs récompenses), une centaine de concerts par an et plusieurs tournées à l'étranger sont au palmarès de l'Orchestre I Musici.

En 2011, il a créé 1'Orchestre Nouvelle Génération formé de ses élèves et de ceux de sa femme. Yuli Turovsky a mis un terme à ses activités de chef d'orchestre et de concertiste en 2011 pour des raisons de santé, mais il continue d'enseigner à la Faculté de musique. Sa carrière et sa contribution à l'essor de la culture musicale canadienne ont été couronnées par deux distinctions honorifiques : il a été nommé chevalier de l'Ordre national du Québec, en 2010, et officier de l'Ordre du Canada en 2011. 
1'Université de Montréal, soliste et chef d'orchestre ${ }^{13}$. Les témoignages recueillis ont mis en évidence l'impact psychologique et effectif que peut avoir un enseignant sur la formation et la professionnalisation de ces jeunes musiciens.

Les questions posées aux participants concernaient leur parcours musical, l'enseignement reçu, leur définition du professionnel en musique ainsi que leurs visées professionnelles. L'analyse des données collectées a fait apparaître le concept de modèle comme facteur déterminant dans la motivation et la formation des étudiants, et comme voie d'accès au milieu professionnel. Cela m'a permis d'élaborer l'hypothèse suivante : dans un domaine où le passage du statut d'étudiant à celui de professionnel rencontre plusieurs contingences par-delà le diplôme ${ }^{14}$ ou le parcours conventionnel, le professeur, en tant que maître où s'exerce un modèle de référence, établit un lien tangible entre le monde de l'apprentissage et le monde professionnel.

Les prochaines lignes s'arrêteront sur la façon dont la référence au modèle, ou son application, génère et active le lien entre la formation et la professionnalisation des jeunes interprètes. Le concept de modèle sera observé et analysé sous deux aspects : le professeur représente un modèle de référence pour les étudiants ; le professeur utilise le principe du modèle pour créer un pont entre la formation et la professionnalisation.

\section{LE MONDE DE L'APPRENTISSAGE}

\section{Les trois étapes de l'apprentissage}

Dans la foulée des travaux d'Izabela Wagner (2004), on peut distinguer trois étapes essentielles dans la formation des futurs musiciens professionnels. Tout d'abord, au début de l'apprentissage, la collaboration qui s'instaure entre les parents, le professeur et l'élève. Lorsqu'il est possible, le suivi des parents dans le travail quotidien du jeune étudiant est un soutien précieux qui peut contribuer à maintenir la motivation de l'enfant et à l'aider à accepter les contraintes et les exigences liées à l'apprentissage.

Durant ses premières années de formation, Stéphane Tétreault a reçu l'aide de son père, musicien amateur, qui a su trouver des moyens appropriés pour stimuler et encourager le travail quotidien de l'enfant :

Lorsque j'ai débuté avec monsieur Turovsky, il me donnait trente études à travailler sur deux semaines. C'est beaucoup pour un enfant de neuf ans, mais heureusement mon père avait une bonne tactique. Il prenait sa guitare, me jouait les études puis nous les jouions ensuite ensemble. C'était motivant.

13 Malgré un contact établi avec sa fille durant neuf mois, il a été impossible de réaliser une entrevue avec Yuli Turovsky, en raison de son état de santé précaire. De plus, le décès subit de son épouse, en mars 2012, a également fortement compromis les possibilités de l'approcher.

14 Comme le souligne Menger: «Dans un univers professionnel comme celui des métiers du spectacle, les traditionnels signaux institutionnels (les diplômes) n'ont pas la valeur habituelle, ne serait-ce qu'en raison de la nature même du travail accompli, qui exige [...] une grande variété d'aptitudes » (Menger 2005, p. 58). 
Plus tard, ayant compris l'utilité du travail régulier, Stéphane a trouvé en lui les ressources nécessaires pour le réaliser.

La seconde étape apparaît au moment où l'étudiant a déjà acquis une certaine autonomie et où il choisit, soit son établissement d'enseignement, soit le professeur avec qui il va poursuivre - et souvent terminer - sa formation ; ce professeur agit fréquemment comme un modèle pour le jeune interprète.

La troisième étape, que Wagner nomme «l'affranchissement partiel du réseau de soutien initial ", représente l'émancipation progressive de la tutelle professorale et l'accès graduel au milieu professionnel. La présente étude s'intéresse plus particulièrement au passage entre la deuxième et la troisième étape afin d'observer l'impact du rôle et des actions du professeur dans ce moment charnière de la carrière d'un jeune interprète.

\section{Le professeur : Yuli Turovsky}

Yuli Turovsky est une figure marquante du milieu musical canadien. Musicien immigrant, son parcours professionnel et sa renommée lui ont permis d'occuper une position stratégique et influente sur la scène musicale québécoise. Parallèlement à ses activités d'enseignant, il a mené une carrière internationale de soliste, de musicien de chambre et de chef d'orchestre. Cette constante immersion dans le milieu professionnel lui a procuré une connaissance des attentes des diffuseurs et du public, ainsi que des difficultés que peut rencontrer un interprète tout au long de sa carrière.

Dans cette optique, c'est un professeur particulièrement apte à guider de jeunes musiciens sur la voie de la professionnalisation, et il a entrepris à cet effet plusieurs démarches importantes qui le distinguent par rapport à d'autres enseignants de la Faculté de musique de 1'Université de Montréal : fondation de deux orchestres de chambre avec ses étudiants et ceux de sa femme ${ }^{15}$ (I Musici de Montréal ${ }^{16}$, en 1983, Orchestre Nouvelle Génération ${ }^{17}$, en 2011) ; mise en place du Concours international pour jeunes solistes en formation lorsqu'il était directeur artistique du Centre d'arts Orford, entre 1995 et 1999. Plusieurs actions qui ont permis d'établir des ponts entre le monde de l'apprentissage et le milieu professionnel, comblant ainsi le manque de filières institutionnelles.

15 Eleonora Turovsky (1939-2012) a été professeure de violon à l'Université de Montréal de 1991 à 2012.

16 Orchestre formé en 1983 par Yuli Turovsky avec des étudiants de sa classe du conservatoire et des étudiants de sa femme, Eleonora. Yuli Turovsky a dirigé cet orchestre jusqu'en 2011, cédant sa place à Jean-Marie Zeitouni. Pour plus d'informations sur cet orchestre, consulter le site : http://imusici.com (consulté le 17 février 2012).

17 Ne dirigeant plus, pour des raisons de santé, Yuli Turovsky est directeur artistique de l'Orchestre Nouvelle Génération. Pour plus d'informations sur cet orchestre, voir le site : http://orchestrenouvellegeneration.com/?lang=fr (consulté le 17 février 2012). 


\section{Les étudiants}

Les trois étudiants retenus pour cette recherche présentent trois niveaux d'études, trois trajectoires musicales et trois cheminements personnels différents ; ils poursuivent toutefois le même objectif : devenir des musiciens professionnels. Ces participants mettent en scène trois cas types d'étudiants impliqués dans un cursus d'enseignement et de professionnalisation, et leurs témoignages m'ont permis d'évaluer la nature et la portée du rôle joué par Yuli Turovsky auprès des jeunes interprètes de la Faculté de musique.

Jusqu'à ses études universitaires, Zhou Fang, a suivi un cursus de formation intégrant l'enseignement scolaire général avec l'enseignement musical. Elle se sent très à l'aise dans un orchestre et souhaite obtenir un poste de musicienne d'orchestre, ce métier procurant plus de sécurité à un interprète qu'une carrière de soliste. Elle est élève de Yuli Turovsky depuis deux ans. Pour elle, la méthode d'enseignement de Yuli Turovsky apporte des solutions à certaines questions qu'elle se posait par rapport à son jeu instrumental et auxquelles aucun de ses précédents professeurs n'avait répondu.

Julie Fuchs a suivi une formation musicale institutionnelle (en France) parallèlement à un cursus scolaire général. Par la suite, elle a enrichi son parcours d'interprète avec des études en musicologie, ce qui lui donne le profil d'une musicienne polyvalente. Bien que n'étant pas élève de Yuli Turovsky, elle bénéficie indirectement de son rayonnement en tant que membre de l'Orchestre Nouvelle Génération. Le regard plus distant qu'elle porte sur ce professeur permet de compléter le portrait qu'en dressent les deux autres étudiants. De plus, Julie a connu en France une expérience de professionnalisation conséquente qu'il est intéressant de mettre en parallèle avec celle que vivent les jeunes interprètes au Québec.

La formation de Stéphane Tétreault est entièrement axée sur la carrière de soliste, ce qui a engendré une scolarité générale discontinue où la présence en classe alternait avec les cours à domicile. Le but de Stéphane Tétreaut est d'être soliste virtuose, tel qu'il l'a exprimé en entrevue : "Depuis que j'ai neuf ans, j'ai toujours voulu être soliste. Je n'ai jamais été vraiment été intéressé par la profession de musicien d'orchestre. » Il étudie avec Yuli Turovsky depuis l'âge de neuf ans et il s'est instauré entre le professeur et l'élève une complicité et un lien étroit de reconnaissance et de confiance mutuelle.

\section{Le choix du professeur, une étape décisive dans la formation}

Les étudiants interrogés ont unanimement déclaré qu'il était fondamental, durant leur formation musicale, de pouvoir choisir leur professeur : un musicien qu'ils admirent, qui a une réputation établie et, très souvent, avec qui ils ont de grandes affinités esthétiques ${ }^{18}$. Les attentes sont importantes vis-à-vis du professeur, car c'est

18 C'est quelques fois le choix d'un instrument ancien lié à un répertoire particulier (Renaissance, Baroque) qui guidera les étudiants vers tel ou tel professeur spécialiste dans ce domaine. 
lui, en sa qualité de mentor « se souciant de leur réalisation personnelle et professionnelle » (Houde 2012, p. 28), qui va les aider à passer du monde de l'apprentissage à celui de la professionnalisation.

Le choix d'un professeur renommé se fait généralement à un moment où, d'une part, l'étudiant possède un degré de connaissance artistique lui permettant d'évaluer plus précisément ses besoins et ses lacunes en matière de formation et, d'autre part, il estime avoir atteint un niveau technique suffisamment solide pour se présenter devant un " grand personnage ", tant cette image est forte. Tel que l'a exprimé Zhou Fang :

Il faut avoir un certain niveau, être confiant avant d'aller voir un grand personnage ; il faut avoir quelque chose à lui montrer. J'étais très intimidée avant de rencontrer Yuli Turovsky, mais finalement, j'ai réalisé que ces grands personnages ne sont pas si impressionnants, mais plutôt ouverts et compréhensifs.

Le choix d'un professeur réputé représente un certain défi à relever pour l'étudiant. En soumettant à l'évaluation d'un maître reconnu le niveau de compétence et de confiance qu'il a acquis, le jeune interprète obtient des indices qui le conforteront ou non sur ses aptitudes à s'engager dans une carrière professionnelle. Le choix d'un professeur est, pour l'élève, une décision importante dans son parcours d'apprentissage. C'est le début d'une période où l'étudiant prend en main sa formation et où il accepte d'en assumer la responsabilité et les conséquences.

À ce titre, l'exemple de Zhou Fang est assez éloquent : " Jusqu'ici, je n'ai jamais choisi mes professeurs, c'était plus le résultat du hasard. Mais à présent, j'ai besoin de prendre ma vie en main et je veux faire mes propres choix. J'ai choisi d'entrer dans la classe de Yuli Turovsky, car depuis longtemps je désirais étudier avec lui ». Les résultats de ce choix ont eu des conséquences positives pour Zhou Fang qui avoue avoir trouvé, grâce à l'enseignement de ce professeur, un équilibre et une confiance qui lui avaient fait défaut jusqu'ici :

Avant, lorsquejejouais, je me fiais uniquement à mes émotions, j'allais un peu à l'aveuglette, sans rien de rationnel pour supporter tout ça. Monsieur Turovsky me donne des bases techniques pour apprendre à maîtriser tout ça. Il m'aide à faire des liens entre la technique et les émotions. C'est une leçon de vie.

Au cours de l'entrevue, Zhou Fang a évoqué à plusieurs reprises les ruptures qui se sont produites dans son parcours musical et ses doutes liés au choix d'une carrière qui avait été élaborée en dehors d'elle (par ses proches). Cette incertitude s'est manifestée à la fois par les diverses incursions de l'étudiante dans des domaines de formation autres que la musique, et par le sentiment d'avoir suivi un parcours où peu de choses étaient sous son contrôle :

J'ai eu des débuts talentueux. Vu de l'extérieur, mon jeu paraissait naturel et assez virtuose. Mais, en moi, comme ma démarche musicale ne venait pas de ma propre initiative, j'avais l'impression que je jouais de façon automatique, sans comprendre vraiment ce que je faisais. C'était plutôt comme si j'étais forcée de le faire. 
Très rapidement, Yuli Turovsky s'est rendu compte des difficultés rencontrées par Zhou Fang et de sa relation en porte-à-faux avec l'instrument. D'un commun accord, l'élève et le professeur ont décidé de reprendre des principes de base (technique, répertoire) afin que Zhou puisse contrôler et maîtriser son jeu instrumental : "Nous travaillons à solidifier ma technique et je me sens beaucoup plus à l'aise. Les choses vont de mieux en mieux et je suis confiante à présent. " Zhou Fang a trouvé en Yuli Turovsky un modèle stabilisant et motivant. Le choix de ce professeur constitue une étape significative dans son cheminement musical.

\section{L'image du professeur}

L'image perçue du professeur est d'autant plus crédible et valorisante que sa reconnaissance sociale et sa réputation d'interprète sont étendues et qu'elles peuvent avoir des retombées sur l'étudiant par le biais d'un transfert de capital social et artistique. Il cherche ainsi à bénéficier de la réputation ${ }^{19}$ de l'enseignant, car elle lui procure une sorte de label de qualité auprès de ses confrères, du public et des employeurs. La réputation possède une dimension évaluatrice qui permet d'obtenir de l'information sur la personne ${ }^{20}$. Elle est vue comme un gage de réussite sociale et artistique. Dans un contexte d'apprentissage, la réputation fonde la relation d'autorité, non sans exercer une forme d'admiration.

Interrogés au sujet de leur professeur, Yuli Turovsky, Stéphane Tétreault et Zhou Fang l'ont ainsi défini : "Il est très impressionnant. Lorsque je suis en train de jouer et qu'il entre dans la pièce, ça change tout, il est inspirant » (Stéphane Tétreault) et «Il est inspirant, libérateur. Je le trouve divin » (Zhou Fang). Quant à Julie Fuchs, n'étant pas directement sous la tutelle de Yuli Turovsky, elle a pu observer le respect et l'admiration que ses élèves lui portent et l'esprit de famille qui caractérise sa classe :

Yuli Turovsky est un rassembleur. C'est quelqu'un de passionné et généreux qui s'implique énormément auprès de ses élèves. Pour moi, c'est une chance immense de pouvoir bénéficier des conseils d'un tel maître grâce à mon appartenance à l'Orchestre Nouvelle Génération.

L'investissement de l'enseignant est perçu par les étudiants comme une preuve de l'intérêt qu'il leur porte et cela contribue à renforcer l'admiration qu'ils éprouvent pour ce maître.

L'image emblématique et charismatique du professeur a des répercussions sur l'estime personnelle de l'étudiant. À propos de Tasso Adamopoulos, Julie Fuchs mentionne que pour elle : "C'était un honneur d'être son élève. Il restera toujours mon maître. » Si le maître accepte de transmettre son savoir à un apprenti, c'est qu'il

19 La réputation est un mécanisme de sélection (au sein des mondes de l'art) découlant d'un processus social qui vise à conférer à l'artiste une valeur particulière. Voir à ce sujet l'analyse proposée par Howard S. Becker ([1982]1988, p. 348-354).

20 Comme le mentionne Menger : «La réputation informe les employeurs sur les aptitudes et sur la valeur du personnel artistique et technique disponible » (Menger 2005, p. 58). 
voit en lui certaines qualités. Ainsi estimé, l'élève gagne en confiance. La transmission peut alors se faire sur un terrain plus fertile, et l'enseignement portera ses fruits.

Le professeur comme modèle

\section{Exemple audio 1: Stéphane Tétreault. Écouter.}

Cristalliser ses aspirations sur un parangon, c'est en quelque sorte établir une certitude quant à la finalité d'une démarche dont la réussite demeure indéterminée. C'est une attitude commune aux trois étudiants interrogés, particulièrement à ce stade de leur formation.

Les archétypes de réussite que concrétisent les artistes consacrés encouragent les jeunes interprètes à tenter leur chance.

\section{Exemple audio 2: Stéphane Tétreault. Écouter.}

Ils sont la preuve tangible que, malgré tous les aléas présents dans le parcours à suivre, la réussite professionnelle est possible, au-delà des difficultés rencontrées. Pour les étudiants, le professeur, c'est la figure emblématique, l'image de l'accomplissement de soi et de la réussite, ce qui est proche d'une forme de mandarinat ${ }^{21}$. Julie Fuchs avoue avoir ressenti le besoin, au cours de sa formation, de vouer une admiration profonde et sincère au professeur avec qui elle travaille. Cette référence à l'image forte et positive d'un enseignant a sans doute contribué à l'encourager et à la guider tout au long de son apprentissage, car dès ses débuts précoces, elle a assumé seule la charge et la responsabilité d'un travail quotidien, ses parents non musiciens n'ayant pu la guider.

Lorsque l'étudiant arrive au stade de sa formation où il entrevoit les difficultés liées à la réalisation d'une carrière artistique, la référence à un modèle lui permet de dépasser les appréhensions ressenties face aux incertitudes de l'avenir professionnel, et apporte une réponse concrète aux questions qu'il se pose et aux doutes qui l'habitent : "C'est rassurant d'avoir des références quand on est étudiant », affirme Julie Fuchs. Car, aux yeux de l'étudiant, si le professeur a réussi, non seulement en tant qu'enseignant mais surtout en tant qu'artiste, c'est qu'il a employé des méthodes appropriées et efficaces, celles qui ont fait leurs preuves. En suivant son enseignement, les jeunes interprètes aspirent à une forme de duplication et espèrent acquérir les outils qui ont permis l'accès à une position privilégiée.

$\mathrm{Au}$ cours du processus de formation puis de professionnalisation, l'identité artistique de l'étudiant se construit. Comme le précise Maryvonne Sorel : « La professionnalisation des individus se joue tout autant au plan des améliorations de connaissances/postures/activités cognitives qu'au plan de l'émotionnalité, des affects ou de

21 On peut, jusqu'à un certain point, rapprocher cette image du professeur de celle du maître dans les musiques traditionnelles non occidentales. Tous deux détenteurs d'un savoir et d'une tradition, possédant un très haut niveau de maîtrise de leur instrument, ils ont atteint une notoriété qui fait d'eux des références en matière d'enseignement et d'interprétation. 
la construction identitaire »(Sorel 2008, p. 44). Dans le processus de construction identitaire, la référence à un modèle est une étape incontournable qu'on peut résumer ainsi : "Un individu [...] trouve ses modèles dans son environnement social [...], il s'agit là d'une identification personnalisée » (Mucchieli 2009, p. 63-64).

L'âge et la maturité de l'étudiant ont une incidence sur le choix de ses modèles de référence. Évoquant son professeur au Conservatoire national supérieur de Lyon, Julie Fuchs reconnaît que Tasso Adamopoulos a agi comme une sorte d'exemple vers qui elle voulait vraiment aller : " Il est la figure la plus marquante de mon parcours musical. Quand j'étais gamine je le voyais déjà dans des magazines musicaux, et je 1'admirais. "Si Julie Fuchs a eu comme modèle Tasso Adamopoulos depuis son enfance, parvenue à présent à l'âge où elle entrevoit les difficultés que peuvent rencontrer les femmes qui désirent concilier maternité, vie familiale et carrière artistique, ses références s'orientent vers des modèles féminins et plus particulièrement vers son actuelle professeure d'alto, Jutta Puchhammer : «Pour moi, Jutta est un exemple de féminité, de réussite et d'équilibre de vie. Par ce qu'elle est et par ce qu'elle fait, elle représente ce à quoi je souhaiterais arriver, car elle fait tout avec bonheur. »

Dans un domaine où le statut professionnel n'est pas totalement circonscrit, la référence au modèle, et le désir de le reproduire, offrent une perspective sécurisante aux apprenants. Cette attitude leur permet de stabiliser leurs objectifs et agit comme une forme de contrepoids à la précarité d'une réussite professionnelle. Cependant, « la relation mentorale est temporaire et non permanente " (Houde 2010, p. 27).

Exemple audio 3 : Julie Fuchs. Écouter.

Afin de s'orienter vers la vie professionnelle, l'étudiant devra progressivement s'affranchir de la tutelle professorale.

\section{AU SEUIL DE LA PROFESSIONNALISATION}

Il s'agit ici de la troisième étape identifiée par Izabela Wagner. Une étape charnière dans le parcours de l'étudiant qui lui permet d'évaluer ses facultés d'adaptation au monde professionnel ainsi que le niveau des compétences accumulées.

\section{Du modèle à l'autonomie}

Stéphane Tétreault et Zhou Fang sont unanimes pour reconnaître que l'approche pédagogique de Yuli Turovsky les incite à réfléchir sur ce qu'ils font, sur ce qu'ils sont, et les amène progressivement à devenir autonomes.

\section{Exemple audio 4 : Zhou Fang. Écouter.}

Il leur donne des moyens pour trouver par eux-mêmes des solutions aux problèmes qu'ils rencontrent et qu'ils rencontreront en tant qu'interprètes s'orientant vers une carrière professionnelle. Le témoignage de Stéphane Tétreault nous permet d'entrevoir comment, à travers l'enseignement dispensé par Yuli Turovsky, l'étudiant acquiert peu à peu son autonomie : "Au début il me montrait avec son violoncelle ; c'est bon d'avoir un exemple parce que tu peux imiter. Puis, quand j'ai eu 12 ans, il ne le 
prenait plus. Il suffisait qu'il dise et puis je trouvais, par moi-même, la façon de faire au violoncelle ce qu'il voulait entendre. "

Comme le mentionne Zhou Fang : "Monsieur Turovsky nous donne des outils, puis ensuite ça nous laisse la liberté d'exprimer ce qu'on ressent. Il n'impose rien. » À un stade où la personnalité artistique de l'étudiant se dessine et s'affirme, il est primordial que l'enseignant fasse confiance à ses capacités expressives et l'aide à les réaliser.

\section{Exemple audio 5 : Stéphane Tétreault. Écouter.}

L'attitude uniquement réceptive de l'élève vis-à-vis de l'enseignant, qui caractérisait ses premières années d'apprentissage, s'est transformée en une relation interactive, une collaboration et un partage des savoirs (Wagner 2004). Pour Julie Fuchs : "Pouvoir partager et échanger des idées avec le professeur, c'est important. Les cours ne doivent pas être unidirectionnels. " Les échanges s'opérant entre l'enseignant et l'étudiant informent ce dernier sur son degré de maturité artistique et sur la validité de ses opinions.

Au seuil de la professionnalisation, l'étudiant doit non seulement acquérir son indépendance, mais également multiplier les expériences professionnelles en dehors de l'institution d'enseignement. D'après les propos des étudiants interrogés, il s'avère que chez les nouvelles générations d'interprètes la césure entre l'enseignement institutionnel et la professionnalisation est quasi absente. Il existe ainsi une forme de porosité entre les deux mondes. Les expériences professionnelles sont parallèles à l'apprentissage dès le niveau du baccalauréat pour une grande partie des jeunes interprètes. D'où l'importance, pour eux, de pouvoir vivre des expériences de scène assez tôt dans leur cursus de formation, et d'acquérir une visibilité qui ira en s'accroissant proportionnellement à l'étendue et aux impacts de leur champ d'action.

\section{Créer un lien}

Le professeur établit non seulement un lien virtuel en tant qu'image de référence vers laquelle tendent les étudiants, mais il instaure également un lien effectif, concret, par les gestes qu'il pose pour contribuer à la professionnalisation des jeunes musiciens.

L'importance de développer des réflexes de scène et d'acquérir rapidement de la visibilité dans le milieu professionnel est une des préoccupations majeures que les enseignants transmettent à leurs élèves. À travers l'analyse des entrevues réalisées, il ressort que le discours du professeur concernant le fonctionnement du milieu musical professionnel est pratiquement inexistant. La prépondérance de la pratique sur la théorie, dans la formation et l'exercice du métier d'interprète, rend tout discours inefficace face à la pertinence d'une expérience de scène. La multiplication des expériences professionnelles permet à l'étudiant non seulement d'acquérir du métier, mais aussi d'apprendre à se connaître, à connaître ses limites, à mesurer ses aptitudes (François 2009, p. 7).

En tant que mentor, le professeur est conscient des difficultés du métier. C'est pourquoi il propose des défis aux étudiants tout en leur apportant le soutien nécessaire à leur réalisation. La position occupée par le professeur est alors 
déterminante. Le poids de sa notoriété lui permettra de proposer les meilleurs postes à ses élèves. Ici encore, la notion de réputation entre en jeu, que ce soit celle des orchestres dans lesquels l'étudiant fait des remplacements, celle des chefs qui les dirigent, ou celle des lieux où il se produit. Le bénéfice qu'en retirera le jeune musicien sera d'autant plus crédible qu'il lui permettra d'augmenter sa visibilité et d'étoffer son curriculum vitae.

\section{Exemple audio 6: Julie Fuchs. Écouter.}

Yuli Turovsky est un des rares professeurs de la Faculté de musique qui organise des auditions mensuelles de sa classe. Ces auditions sont précédées, quelques jours auparavant, d'un concert privé chez les Turovsky, ce qui fait office de répétition générale. Usant également de son influence et de ses relations dans le milieu musical montréalais, Yuli Turovsky présente régulièrement ses étudiants les plus avancés à divers organisateurs de concerts ou de festivals, afin qu'ils se produisent en dehors du cadre scolaire. Il a aussi fréquemment fait appel à certains de ses étudiants pour faire des remplacements dans 1'Orchestre I Musici ou pour se produire en soliste avec cet orchestre, tel Stéphane Tétreault qui a joué sous la direction de Maxime Vengerov en octobre 2010.

Parmi les actions entreprises par Yuli Turovsky pour établir des liens entre le monde de l'apprentissage et le milieu professionnel, une des plus importantes a été la création de deux orchestres de chambre avec ses étudiants et ceux de sa défunte femme : I Musici de Montréal, en 1983, et l'Orchestre Nouvelle Génération, en 2011. Si I Musici réunissait de jeunes musiciens ayant terminé leur cursus de formation, l'Orchestre Nouvelle Génération est essentiellement composé d'étudiants présentement inscrits dans les classes de Yuli et Eleonora Turovsky à la Faculté de musique de l'Université de Montréal. Conscient que, dans le contexte musical actuel, le développement de stratégies d'adaptation est plus précoce chez les nouvelles générations de musiciens que chez leurs aînés ${ }^{22}$, Yuli Turovsky a mis en place une structure d'encadrement qui fait office de tremplin pour la professionnalisation des jeunes apprenants. Une démarche d'autant plus pertinente au Québec où la multiplicité des emplois intermittents l'emporte sur celle des emplois dans les structures stables.

Bénéficiant de la réputation de l'Orchestre I Musici et de celle de Yuli Turovsky, l'Orchestre Nouvelle Génération réunit toutes les conditions d'exercice du métier dans un cadre professionnel structuré : saison de concerts (quatre concerts à thématiques variées, en collaboration avec des acteurs et metteurs en scène professionnels) dans

22 Concernant les conditions actuelles d'accès au milieu musical professionnel québécois, on observe certains changements par rapport au cheminement de musiciens en poste depuis une vingtaine d'années. Pour ces derniers, les premières expériences de scène se déroulaient essentiellement dans le cadre des institutions d'enseignement. La frontière entre le statut d'étudiant et celui de professionnel était relativement marquée. Une fois les études terminées, ces musiciens trouvaient rapidement, soit un emploi dans un orchestre (avec un statut de permanent, ou de surnuméraire), soit un poste d'enseignant qui leur permettait de développer parallèlement une carrière d'interprète. (Ces informations ont été recueillies auprès de musiciens actifs dans le milieu musical québécois depuis une vingtaine d'années, dans le cadre de ma recherche de maîtrise réalisée à l'Université de Montréal de 2010 à 2011.) 
un cadre institutionnel (salle Claude-Champagne de l'Université de Montréal) ; trois chefs attitrés ; formule d'abonnements ; conseil d'administration ; siège social ; sponsors et campagnes de publicité. Cet orchestre procure aux étudiants une forme de continuité et de permanence dans leur cursus de professionnalisation.

Cependant, si les expériences en terrain professionnel sont formatrices et offrent une rémunération, elles ne sont pas toutes valorisantes. Jouer dans le cadre d'une cérémonie religieuse, participer à un événement communautaire ou culturel, ou même, jouer dans le métro : autant de situations qui peuvent permettre aux étudiants d'acquérir du métier, de gagner de l'argent, mais qui n'apporteront pas le prestige d'une prestation réalisée sur une scène de concert ou avec un orchestre renommé : "Lorsque j'étais dans l'Orchestre Gustav Mahler, j'ai voyagé dans toute l'Europe, j'ai joué dans plusieurs grandes salles - notamment à Bayreuth - c'est une chance inouïe. C'est formateur, car en même temps, c'est mon futur métier » (Julie Fuchs).

Concernant les expériences transitoires, celles qui remplissent l'espace entre deux états, le témoignage et le parcours de Julie Fuchs ont permis d'observer des différences frappantes entre le contexte musical québécois et celui de la France. En comparant les expériences en milieu professionnel qu'elle a connues en France avec celles qu'elle expérimente au Québec, Julie Fuchs a fait les constatations suivantes : au Québec, les opportunités de remplacement dans des orchestres sont peu nombreuses pour les jeunes interprètes (moins d'orchestres nationaux et de maisons d'opéra qu'en France) ; par contre, il y a davantage de possibilités d'obtenir de petits engagements dans plusieurs secteurs (évènements culturels, célébrations religieuses, création de spectacles et de projets personnels). Toutefois, au Québec, les conditions de travail sont beaucoup moins structurées et formalisées qu'en France (contrats, congés payés, frais de déplacement, régime d'intermittence, caisse de retraite).

Ces divergences influent sur les conditions de professionnalisation des jeunes interprètes. Alors que le contexte musical français permet encore de songer à une carrière stable et traditionnelle de musicien d'orchestre, même en surnuméraire, grâce au statut d'intermittent du spectacle, au Québec une telle carrière est difficilement envisageable, car réservée à un nombre très restreint de musiciens qui, de plus, entrent en compétition avec des musiciens étrangers qui concourent pour les postes d'orchestre.

C'est ici que l'on peut mesurer la pertinence des actions de Yuli Turovsky, en concomitance avec les retombées qu'engendre la présence de ses élèves dans le milieu artistique québécois. Usant de sa position privilégiée, il est le mandataire de ses étudiants auprès des diffuseurs, et sa réputation, gage de qualité, leur ouvre des portes sur la scène musicale. Il contribue ainsi à la socialisation de ses élèves après les avoir formés. Ces expériences de scène sont très appréciées des étudiants. En effet, elles sont pour eux formatrices et stimulantes, l'expérience étant le « moteur du développement des compétences»(Champy-Remoussenard 2008, p. 76). Aussi, elles contribuent à consolider la confiance des apprenants et les incitent à se dépasser, tout en posant des jalons pour leur future carrière ${ }^{23}$.

23 L'exemple de Stéphane Tétreault est à ce titre significatif. Formé et guidé par Yuli Turovsky, il multiplie les participations aux concours nationaux et internationaux depuis plusieurs années. 
En se faisant les répondants des interprètes qu'ils ont formés, des professeurs comme Yuli Turovsky procèdent à une forme de transfert de crédibilité tout en contribuant à faire de leurs étudiants des musiciens professionnels ${ }^{24}$. Ils cherchent à placer leurs disciples à des postes stratégiques au sein du milieu musical, opérant ainsi une forme de contrôle des marchés artistiques. Cela a pour but de perpétuer une tradition, de renouveler la culture et de garder en vie l'organisation et ses membres (Houde 2010, p. 10).

\section{Reproduire un modèle et perpétuer une tradition}

En fondant l'Orchestre I Musici de Montréal, Yuli Turovsky reprenait à son compte un modèle d'orchestre de chambre éprouvé, tel qu'il l'a connu en Russie (Orchestre de chambre de Moscou), puis en Italie (I Musici di Roma), où il a séjourné huit mois avant d'immigrer au Québec. Les succès rencontrés par l'Orchestre I Musici de Montréal, ainsi que sa durabilité, prouvent la fiabilité d'une telle structure. L'Orchestre Nouvelle Génération est ainsi en grande partie calqué sur 1'Orchestre I Musici (nombre de musiciens, répertoire, esthétique musicale). La reproduction d'un modèle dont on connaît les paramètres et le mode de fonctionnement permet d'établir une voie d'accès au milieu professionnel plus fiable et plus contrôlable, en bénéficiant de l'expérience acquise par le modèle reproduit.

Une autre facette du modèle utilisé par Yuli Turovsky est l'implantation d'une école d'interprétation. L'Orchestre Nouvelle Génération n'a pas pour unique mission d'établir un pont entre le milieu de l'apprentissage et le monde professionnel. Un des autres buts visés par Yuli Turovsky est celui « d'assurer que l'apprentissage, la croissance, l'assimilation et la continuation des principes artistiques d'une école distincte de performance de musique de chambre établis par Yuli Turovsky soient perpétués ${ }^{25} »$.

Il faut noter la singularité de la démarche de Yuli Turovsky qui, en tant que musicien étranger, a implanté ses paramètres musicaux au Québec par le biais des orchestres qu'il a fondés avec ses étudiants, et à travers son enseignement. L'école stylistique qui découle de ses efforts allie les principes de l'école russe de violoncelle à ceux que le musicien a développés tout au long de sa carrière, en liaison avec sa personnalité et sa sensibilité. La perpétuation d'une tradition influente (école russe de cordes),

Les retombées de ces concours se concrétisent par les engagements qu'il obtient pour participer à plusieurs festivals ou pour jouer, en soliste, avec des orchestres ou des ensembles professionnels (Orchestre Métropolitain, Nouvel Ensemble Moderne, Orchestre I Musici de Montréal, Orchestre Philarmonique de Malaisie, etc.).

24 À titre d'exemple, on peut citer quelques anciens élèves de Yuli Turovsky qui occupent, à présent, une position de choix dans le milieu musical montréalais : Yegor Dyachkov (concertiste international et professeur à la Faculté de musique de l'Université de Montréal et à l'Université McGill) ; Velitchka Yotcheva (concertiste internationale, fondatrice et directrice artistique de la Société de Musique de Chambre de Montréal) ou Luo Di (membre du trio Muczynsky, ensemble qui a été finaliste et lauréat de plusieurs concours internationaux).

25 Voir : www.orchestrenouvellegeneration.com (consulté le 10 mars 2012). 
dont on peut retracer l'histoire et dont la renommée de ses représentants en assure la réputation ${ }^{26}$, peut constituer un gage de réussite à 1'accès à la profession pour les étudiants issus de cette école. Car la stabilité et la renommée d'une école d'interprétation établissent des référents permettant à la communauté musicienne d'identifier et de cataloguer les musiciens qui en sont issus.

La pérennité d'une école stylistique d'interprétation est en partie assurée par la filiation qui existe entre un maître et ses élèves. Parmi les étudiants de Yuli Turovsky, Stéphane Tétreault présente des dispositions particulières qui le distinguent de ses congénères. Le professeur entrevoit en lui un possible successeur vers lequel il peut transférer une partie de son patrimoine musical afin de maintenir la continuité des connaissances accumulées. Yuli Turovsky mise sur un élève dont il perçoit le potentiel artistique, son jugement étant validé par un " faisceau de signaux » le renseignant sur les chances de réussite de l'étudiant (François 2009, p. 8). Les engagements professionnels de Stéphane Tétreault, ses résultats aux concours internationaux, sa présence dans les médias et le fait qu'il soit le dépositaire d'un instrument de grande valeur sont autant de gages des qualités et du mérite du jeune interprète. En transmettant à Stéphane Tétreault son savoir (Yuli Turovsky l'a également formé à la direction d'orchestre) et son héritage musical ${ }^{27}$, il procède à un transfert de capital social et artistique.

\section{CONCLUSION}

Le déphasage existant entre l'apprentissage et l'exercice du métier complexifie l'insertion professionnelle des jeunes interprètes. En l'absence d'un lien institutionnel entre la formation et le milieu professionnel, la relation d'individu à individu est décisive dans le processus de professionnalisation des jeunes musiciens. Le professeur, grâce à la position qu'il occupe dans le milieu musical, et par les actions qu'il entreprend pour ses étudiants, contribue à instituer un lien essentiel entre le monde de l'apprentissage et le monde professionnel.

La référence à la tradition historique dans l'enseignement musical institutionnel demeure forte. Il y a une propension chez les interprètes à perpétuer l'image du modèle, et à reproduire les stratégies et les mécanismes d'intégration professionnelle déjà éprouvés. Tant que les modèles représentés par les professeurs en poste subsisteront, ce type de cheminement, qui est en apparence efficace, servira d'exemple aux apprenants. La reproduction d'un modèle permet de contrer partiellement le hasard qui entoure la réalisation d'une carrière professionnelle, carrière dont le parcours est affecté par plusieurs variables : incertitude de réussite, forte concurrence, fluctuations des tendances artistiques et commerciales, appréciation du public, etc.

26 À ce titre, on peut mentionner la notoriété dont a bénéficié l'école de cordes russe en Amérique du Nord suite à l'immigration de nombreux interprètes russes aux États-Unis, dans la première moitié du $\mathrm{xx}^{\mathrm{e}}$ siècle.

27 En entrevue, Stéphane m'a confié qu'il se situait dans la filiation de l'école de violoncelle russe dont est issu Yuli Turovsky, l'instigateur de cette école étant Karl Davidov. 
En appliquant les principes de modèles efficients (orchestre de chambre et école stylistique d'interprétation), Yuli Turovsky permet à ses étudiants d'emprunter la filière qu'il a tracée quelques années auparavant pour accéder au milieu musical québécois. Le modèle agit alors comme un outil d'insertion professionnelle. S'appuyant sur la stabilité qu'offrent des modèles reconnus, Yuli Turovsky établit un lien entre le milieu institutionnel et le milieu professionnel, balisant pour ses étudiants une voie vers la professionnalisation.

Ainsi, dans un milieu très concurrentiel et compétitif, un personnage réputé occupant une position stratégique " utilise son expertise [...] et ses compétences de façon créatrice et productive " (Houde 2010, p. 36), pour permettre aux jeunes interprètes d'accéder à la profession. Cette situation donne lieu à une forme de mentorat indispensable à la survie et à la transmission d'un savoir et d'une tradition, ce qui est la mission de l'Orchestre Nouvelle Génération.

\section{BIBLIOGRAPHIE}

Becker, Howard S. ([1982]1988), Les mondes de l'art, présentation de Pierre-Michel Menger, traduit par Jeanne Bouniort, Paris, Flammarion.

Champy-Remoussard, Patricia (2008), "Incontournable professionnalisation ", Savoirs, revue internationale de recherche en éducation et formation des adultes, $\mathrm{n}^{\circ}$ 17, p. 51-61.

Conseil des arts et des lettres du Québec (CALQ) (2012-2013), " Musique. Artiste professionnel ", www.calq.gouv.qc.ca/artistes/musique.htm, consulté le 6 juin 2012.

Coulangeon, Philippe (2004), Les musiciens interprètes en France, Paris, La Documentation française.

François, Pierre (2004), " Professionnels et amateurs ", dans Jean-Jacques Nattiez (dir.), Musiques. Une encyclopédie pour le XXI siècle, "Vol. 2. Les savoirs musicaux ", Arles/Paris, Actes Sud/Cité de la musique, p. 585-607.

François, Pierre (2009), «La vocation musicale. De l'illumination individuelle au processus collectif », http://spire.sciencespo.fr/hdl:/2441/f0uohitsgqh8dhk97gcrpe5kk/resources/francois.vocationmusicale.pdf, consulté le 9 novembre 2016.

Guilde des musiciens et musiciennes du Québec (2012), www.gmmq.com, consulté le 17 février 2012.

Houde, Renée (2010), Des mentors pour la relève, Québec, Presses de 1'Université du Québec.

Menger, Pierre-Michel (2005), Les intermittents du spectacle. Sociologie d'une exception, Paris, Éditions de l'École des Hautes études en Sciences sociales.

Menger, Pierre-Michel (2009), Le travail créateur. S'accomplir dans l'incertain, Paris, Seuil/Gallimard.

Mucchielli, Alex (1986), L'identité, Paris, Presses Universitaires de France.

Orchestre de chambre I Musici de Montréal (2012), «L'orchestre », http://imusici.com/, consulté le 10 mars 2012.

Orchestre Nouvelle Génération (2012), http:/ / orchestrenouvellegeneration.com, consulté le 10 mars 2012.

Sorel, Maryvonne (2008), "À propos de la professionnalisation. Le retour du sujet... », Savoirs, revue internationale de recherches en éducation et formation des adultes, $\mathrm{n}^{\circ}$ 17, p. 37-50.

Tafuri, Johannella (2004), "La formation musicale supérieure en Europe et en Amérique du Nord», dans Jean-Jacques Nattiez (dir.), Musiques. Une encyclopédie pour le XXI siècle, « Vol. 2. Les savoirs musicaux », Arles/Paris, Actes Sud/Cité de la musique, p. 873-898.

Union des artistes (2012), https://uda.ca, consulté le 17 février 2012. 
Wagner, Izabela (2004), "La formation des violonistes virtuoses. Les réseaux de soutien ", Sociétés contemporaines, $\mathrm{n}^{\circ}$ 56, p. 133-163, www.cairn.info/revue-societes-contemporaines-2004-4page-133.htm, consulté le 8 septembre 2012. 